\title{
Multiples of Trace Forms and Algebras with Involution
}

\section{Eva Bayer-Fluckiger}

Ecole Polytechnique Fédérale de Lausanne, Mathématiques, CSAG-IMB-FSB, Station 8, 1015 Lausanne, Switzerland

Correspondence to be sent to: eva.bayer@epfl.ch

Let $k$ be a field of characteristic $\neq 2$, and let $G$ be a finite group. The aim of this article is to give a cohomological criterion for the isomorphism of multiples of trace forms of GGalois algebras over $k$. The proof uses results concerning multiples of hermitian forms over division algebras with involution that are of independent interest.

\section{Introduction}

Let $k$ be a field of characteristic $\neq 2$, and let $L$ be a Galois extension of $k$ with group $G$. Let

$$
\begin{gathered}
q_{L}: L \times L \rightarrow k \\
q_{L}(x, y)=\operatorname{Tr}_{L / k}(x y)
\end{gathered}
$$

be the trace form. It is well known that $L$ has a normal basis over $k$, in other words there exists $x \in L$ such that $\{g x\}_{g \in G}$ is a basis of $L$ as a $k$-vector space. Such a basis is called a self-dual normal basis if $q_{L}(g x, h x)=\delta_{g, h}$ for all $g, h \in G$.

The following question was studied in [1], [2], [3], [4], [5], [6]:

QUESTION 1.1. Which Galois extensions have a self-dual normal basis ?

Received July 8, 2007; Revised September 11, 2007; Accepted September 19, 2007

Communicated by Barry Mazur

See http://www.oxfordjournals.org/our_journals/imrn/for proper citation instructions.

(C) The Author 2007. Published by Oxford University Press. All rights reserved. For permissions, please e-mail: journals.permissions@oxfordjournals.org. 
This question is settled in some cases, for instance, when $G$ has odd order [3], when the 2-Sylow subgroups of $G$ are elementary abelian [5], but it is open in general.

Note that the existence of a normal basis is equivalent with the fact that $L$ is a free $k[G]$-module of rank 1. A similar reformulation can be given for the self-dual normal basis question. Indeed, remark that the quadratic form $q_{L}$ is invariant by $G$, that is $q_{L}(g x, g y)=q_{L}(x, y)$ for all $x, y \in L$ and for all $g \in G$. In other words, $q_{L}$ is a $G-$ quadratic form (cf. 2). Let us define the unit G-quadratic form as being $q_{0}: k[G] \times k[G] \rightarrow$ $k$ characterized by $q_{0}(g, h)=\delta_{g, h}$. Then the existence of a self-dual normal basis is equivalent with the isomorphism of $q_{L}$ and $q_{0}$ as $G$-quadratic forms.

It is more natural to work in the category of $G$-Galois algebras instead of Galois extensions with group $G$. Let us denote by $L_{0}$ the split $G-G$ alois algebra; then $q_{L_{0}} \simeq_{G} q_{0}$. This leads us to the following question:

QUESTION 1.2. Let $L$ and $L^{\prime}$ be two G-Galois algebras. When are the G-forms $q_{L}$ and $q_{L^{\prime}}$ isomorphic?

The results of $[3,5]$ apply to this more general situation. However, a complete answer to the question seems out of reach at this point. For this reason, a weaker question was raised in [1]. Indeed, if $\phi$ is a nondegenerate quadratic form and $q$ is a $G$-quadratic form, then the tensor product $\phi \otimes q$ is a $G$-quadratic form. One can ask the following question.

QUESTION 1.3. Let $L$ and $L^{\prime}$ be two G-Galois algebras, and let $\phi$ be a nondegenerate quadratic form. When are the $G$-forms $\phi \otimes q_{L}$ and $\phi \otimes q_{L^{\prime}}$ isomorphic?

If $\phi$ is an odd-dimensional form, then this question is equivalent with the previous one. Let $W(k)$ be the Witt ring of $k$, and let $I$ be the ideal of $W(k)$ consisting of the Witt classes of the even-dimensional forms. Let $k_{s}$ be a separable closure of $k$, and set $\Gamma_{k}=\mathrm{Gal}\left(k_{s} / k\right)$. For every positive integer $n$, let us denote by $e_{n}: I^{n} / I^{n+1} \rightarrow H^{1}\left(\Gamma_{k}, \mathbf{Z} / 2 \mathbf{Z}\right)$ the MilnorVoevodsky isomorphism (see 2). Let $\mathrm{cd}_{2}\left(\Gamma_{k}\right)$ be the 2-cohomological dimension of $\Gamma_{k}$ (cf. 2 ), and let $d \geq 0$ be an integer. The following two statements are easy consequences of the above isomorphisms (cf. 2.2, 2.5):

I. Suppose that $\mathrm{cd}_{2}\left(\Gamma_{k}\right) \leq d$. Let $q$ and $q^{\prime}$ be two quadratic forms with $\operatorname{dim}(q)=\operatorname{dim}\left(q^{\prime}\right)$, and let $\phi \in I^{d}$. Then

$\phi \otimes q \simeq \phi \otimes q^{\prime}$. 
II. Suppose that $\operatorname{cd}_{2}\left(\Gamma_{k}\right) \leq d$. Let $q$ and $q^{\prime}$ be two quadratic forms with $\operatorname{dim}(q)=\operatorname{dim}\left(q^{\prime}\right)$, and let $\phi \in I^{d-1}$. Then

$$
\phi \otimes q \simeq \phi \otimes q^{\prime} \text { if and only if } e_{d-1}(\phi) \cup(\operatorname{disc}(q))=e_{d-1}(\phi) \cup\left(\operatorname{disc}\left(q^{\prime}\right)\right) \text { in } H^{d}(k) .
$$

It is natural to look for similar statements concerning trace forms of $G-G a l o i s ~ a l g e b r a s$, as proposed in [1]. As an analog of $\mathrm{I}$, we have the following :

THEOREM 1.4. (Chabloz, [9]) Suppose that $\mathrm{cd}_{2}\left(\Gamma_{k}\right) \leq d$. Let $L$ and $L^{\prime}$ be two G-Galois algebras, and let $\phi \in I^{d}$. Then $\phi \otimes q_{L} \simeq_{G} \phi \otimes q_{L^{\prime}}$.

In order to go further, we need some invariants defined in [5]. Let $f_{L}: \Gamma_{k} \rightarrow G$ be a continuous homomorphism corresponding to the $G$-Galois algebra $L$. The homomorphism $f_{L}$ induces $f_{L}^{*}: H^{1}(G, \mathbf{Z} / 2 \mathbf{Z}) \rightarrow H^{1}\left(\Gamma_{k}, \mathbf{Z} / 2 \mathbf{Z}\right)$. For all $x \in H^{1}(G, \mathbf{Z} / 2 \mathbf{Z})$, set $x_{L}=f_{L}^{*}(x)$. Then $x_{L}$ is an invariant of the $G$-quadratic form $q_{L}$ (cf. [5], 2.2.3). The following statement is inspired by II, and is proved in Section 4 :

THEOREM 1.5. Suppose that $\operatorname{cd}_{2}\left(\Gamma_{k}\right) \leq d$. Let $L$ and $L^{\prime}$ be two G-Galois algebras, and let $\phi \in I^{d-1}$. Then the $G$-quadratic forms $\phi \otimes q_{L}$ and $\phi \otimes q_{L^{\prime}}$ are isomorphic if and only if $e_{d-1}(\phi) \cup x_{L}=e_{d-1}(\phi) \cup x_{L^{\prime}}$ for all $x \in H^{1}(G, \mathbf{Z} / 2 \mathbf{Z})$.

This was conjectured in [1], and proved in special cases by Chabloz, Monsurro, Morales, Parimala and Schoof (see [4],[9]-[12]). The proof uses results concerning hermitian forms over algebras with involution (see Section 2.5 and Section 3 for details). Let $(D, \sigma)$ be a division algebra with involution over $k$, and let $W(D, \sigma)$ be the Witt group of hermitian forms over $(D, \sigma)$. Then $W(D, \sigma)$ is a $W(k)$-module. Let us denote by $J$ the $W(k)-$ submodule of $W(D, \sigma)$ consisting of even dimensional hermitian forms over $D$.

THEOREM 1.6. Suppose that $\mathrm{cd}_{2}\left(\Gamma_{k}\right) \leq d$. Then

(a) We have $I^{d} J=0$.

(b) If $\sigma$ is of the second kind, then $I^{d-1} J=0$.

(c) If $\sigma$ is of the first kind and of the symplectic type, then $I^{d-2} J=0$.

Part (a) is due to Chabloz [9]. Parts (b) and (c) are proved in Section 3, and are used in the proof of the main result of this article in Section 4. In order to deal with involutions of the first kind and of the orthogonal type, we need the following: 
THEOREM 1.7. Suppose that $\operatorname{cd}_{2}\left(\Gamma_{k}\right) \leq d$. Let $(D, \sigma)$ be a quaternion algebra with an orthogonal involution, and let $(V, h)$ and $\left(V^{\prime}, h^{\prime}\right)$ be two hermitian forms over $(D, \sigma)$ with $\operatorname{dim}_{D}(V)=\operatorname{dim}_{D}\left(V^{\prime}\right)$. Let $\phi \in I^{d-1}$. Then $\phi \otimes h \simeq \phi \otimes h^{\prime}$ if and only if

$$
e_{d-1}(\phi) \cup(\operatorname{disc}(h))=e_{d-1}(\phi) \cup\left(\operatorname{disc}\left(h^{\prime}\right)\right) .
$$

This follows from the results of Parimala, Sridharan and Suresh [14] and of Berhuy [8].

\section{Definitions, Notation and Basic Facts}

\subsection{Galois cohomology}

Let $k_{s}$ be a separable closure of $k$, and set $\Gamma_{k}=\operatorname{Gal}\left(k_{s} / k\right)$. For any discrete $\Gamma_{k}-$ module $C$, set $H^{i}(k, C)=H^{i}\left(\Gamma_{k}, C\right)$. We say that the 2-cohomological dimension of $\Gamma_{k}$ is at most $d$, denoted by $\operatorname{cd}_{2}\left(\Gamma_{k}\right) \leq d$, if $H^{i}(k, C)=0$ for all $i>d$ and for every finite 2-primary $\Gamma_{k}$-module $C$.

Set $H^{i}(k)=H^{i}(k, \mathbf{Z} / 2 \mathbf{Z})$, and recall that $H^{1}(k) \simeq k^{*} / k^{* 2}$. For all $a \in k^{*}$, let us denote by $(a) \in H^{1}(k)$ the corresponding cohomology class. We use the additive notation for $H^{1}(k)$. If $a_{1}, \ldots, a_{n} \in k^{*}$, we denote by $\left(a_{1}\right) \cup \cdots \cup\left(a_{n}\right) \in H^{n}(k)$ their cup product.

If $U$ is a linear algebraic group defined over $k$, let $H^{1}(k, U)$ be the pointed set $H^{1}\left(\Gamma_{k}, U\left(k_{s}\right)\right)$ (cf. $[16,17]$ Ch. 10).

\subsection{Quadratic forms}

All quadratic forms are supposed to be nondegenerate. We denote by $W(k)$ the Witt ring of $k$, and by $I=I(k)$ the fundamental ideal of $W(k)$. For all $a_{1}, \ldots, a_{n} \in k^{*}$, let us denote by $<<a_{1}, \ldots, a_{n}>>=<1,-a_{1}>\otimes \cdots \otimes<1,-a_{n}>$ the associated $n$-fold Pfister form. It is well known that $I^{n}$ is generated by the $n$-fold Pfister forms. The following has been conjectured by Milnor, and proved by Voevodsky (see also Orlov-Vishik-Voevodsky [13][19], and the survey paper [10]) :

THEOREM 2.1 (Voevodsky). For every positive integer $n$, there exists an isomorphism

$$
e_{n}: I^{n} / I^{n+1} \rightarrow H^{n}(k)
$$

such that

$$
e_{n}\left(<<a_{1}, \ldots, a_{n}>>\right)=\left(a_{1}\right) \cup \cdots \cup\left(a_{n}\right)
$$

for all $a_{1}, \ldots, a_{n} \in k^{*}$. 
It is easy to see that the above theorem has the following consequences:

COROLLARY 2.2. Suppose that $\operatorname{cd}_{2}\left(\Gamma_{k}\right) \leq d$. Let $q$ and $q^{\prime}$ be two quadratic forms with $\operatorname{dim}(q)=\operatorname{dim}\left(q^{\prime}\right)$, and let $\phi \in I^{d}$. Then

$\phi \otimes q \simeq \phi \otimes q^{\prime}$

PROOF. Note that by Theorem 2.1, $\operatorname{cd}_{2}\left(\Gamma_{k}\right) \leq d$ implies that $I^{d+1}=0$. As $\operatorname{dim}(q)=\operatorname{dim}\left(q^{\prime}\right)$, we have $q \oplus\left(-q^{\prime}\right) \in I$. Therefore, $\phi \otimes\left(q \oplus\left(-q^{\prime}\right)\right) \in I^{d+1}=0$. This implies that $\phi \otimes q \simeq \phi \otimes q^{\prime}$. For every quadratic form $q$, let us denote by $\operatorname{disc}(q) \in H^{1}(k)$ its discriminant. Recall that if $n=\operatorname{dim}(q)$, then $\operatorname{disc}(q)=(-1)^{\frac{n(n-1)}{2}} \operatorname{det}(q)$. We need the following proposition:

PROPOSITION 2.3. Let $q$ and $q^{\prime}$ be two quadratic forms with $\operatorname{dim}(q)=\operatorname{dim}\left(q^{\prime}\right)$, and let $\phi \in I^{d-1}$. Then

$$
e_{d}\left(\phi \otimes\left(q \oplus\left(-q^{\prime}\right)\right)=e_{d-1}(\phi) \cup(\operatorname{disc}(q))+e_{d-1}(\phi) \cup\left(\operatorname{disc}\left(q^{\prime}\right)\right)\right.
$$

Proof. Set $Q=q \oplus\left(-q^{\prime}\right)$, and let $m=\operatorname{dim}(q)=\operatorname{dim}\left(q^{\prime}\right), n=2 m=\operatorname{dim}(Q)$. Note that $(-1)^{\frac{n(n-1)}{2}}=(-1)^{m}$, hence $(\operatorname{disc}(Q))=(\operatorname{disc}(q))+\left(\operatorname{disc}\left(q^{\prime}\right)\right)$. We have $Q \in I$, and $e_{1}(Q)=(\operatorname{disc}(Q))=(\operatorname{disc}(q))+\left(\operatorname{disc}\left(q^{\prime}\right)\right)$. Therefore,

$$
e_{d}(\phi \otimes Q)=e_{d-1}(\phi) \cup e_{1}(Q)=e_{d-1}(\phi) \cup(\operatorname{disc}(q))+e_{d-1}(\phi) \cup\left(\operatorname{disc}\left(q^{\prime}\right)\right),
$$

and hence the proposition is proved.

COROLLARY 2.4. Let $q$ and $q^{\prime}$ be two quadratic forms with $\operatorname{dim}(q)=\operatorname{dim}\left(q^{\prime}\right)$, and let $\phi \in I^{d-1}$. If $\phi \otimes q \simeq \phi \otimes q^{\prime}$, then $e_{d-1}(\phi) \cup(\operatorname{disc}(q))=e_{d-1}(\phi) \cup\left(\operatorname{disc}\left(q^{\prime}\right)\right) \in H^{d}(k)$.

PRoOF. As $\phi \otimes q \simeq \phi \otimes q^{\prime}$, the quadratic form $\phi\left(\otimes\left(q \oplus\left(-q^{\prime}\right)\right)\right)$ is hyperbolic. Hence $e_{d}\left(\phi \otimes\left(q \oplus\left(-q^{\prime}\right)\right)\right)=0$. By Proposition 2.3, we have

$$
e_{d}\left(\phi \otimes\left(q \oplus\left(-q^{\prime}\right)\right)\right)=e_{d-1}(\phi) \cup(\operatorname{disc}(q))+e_{d-1}(\phi) \cup\left(\operatorname{disc}\left(q^{\prime}\right)\right),
$$

therefore $e_{d-1}(\phi) \cup(\operatorname{disc}(q))=e_{d-1}(\phi) \cup\left(\operatorname{disc}\left(q^{\prime}\right)\right)$, as claimed.

COROLLARY 2.5. Suppose that $\operatorname{cd}_{2}\left(\Gamma_{k}\right) \leq d$. Let $q$ and $q^{\prime}$ be two quadratic forms with $\operatorname{dim}(q)=\operatorname{dim}\left(q^{\prime}\right)$, and let $\phi \in I^{d-1}$. Then $\phi \otimes q \simeq \phi \otimes q^{\prime}$ if and only if $e_{d-1}(\phi) \cup(\operatorname{disc}(q))=$ $e_{d-1}(\phi) \cup\left(\operatorname{disc}\left(q^{\prime}\right)\right) \in H^{d}(k)$. 
ProOF. Set $Q=q \oplus\left(-q^{\prime}\right)$. By Proposition 2.3, we have

$$
e_{d}(\phi \otimes Q)=e_{d-1}(\phi) \cup(\operatorname{disc}(q))+e_{d-1}(\phi) \cup\left(\operatorname{disc}\left(q^{\prime}\right)\right)
$$

Hence $e_{d}(\phi \otimes Q)=0$ is equivalent to $e_{d-1}(\phi) \cup(\operatorname{disc}(q))=e_{d-1}(\phi) \cup\left(\operatorname{disc}\left(q^{\prime}\right)\right)$. But by Theorem 2.1, $e_{d}(\phi \otimes Q)=0$ is equivalent to $\phi \otimes Q$ hyperbolic, hence to $\phi \otimes q \simeq \phi \otimes q^{\prime}$.

\subsection{G-quadratic forms}

Let $G$ be a finite group, and let us denote by $k[G]$ the associated group ring. A $G$-quadratic form is a pair $(M, q)$, where $M$ is a $k[G]$-module that is a finite dimensional $k$-vector space, and $q: M \times M \rightarrow k$ is a nondegenerate symmetric bilinear form such that

$$
q(g x, g y)=q(x, y)
$$

for all $x, y \in M$ and all $g \in G$. We say that two $G$-quadratic forms $(M, q)$ and $\left(M^{\prime}, q^{\prime}\right)$ are isomorphic if there exists an isomorphism of $k[G]$-modules $f: M \rightarrow M^{\prime}$ such that $q(f(x), f(y))=q^{\prime}(x, y)$ for all $x, y \in M$. If this is the case, we write $(M, q) \simeq_{G}\left(M^{\prime}, q^{\prime}\right)$, or $q \simeq_{G} q^{\prime}$. If $\phi$ is a quadratic form over $k$, and $q$ a $G$-quadratic form, then the tensor product $\phi \otimes q$ is a $G$-quadratic form.

\subsection{Trace forms}

Let $L$ be a $G-$ Galois algebra, and let

$$
q_{L}: L \times L \rightarrow k, q_{L}(x, y)=\operatorname{Tr}_{L / k}(x y)
$$

be its trace form. Then $q_{L}$ is a $G$-quadratic form. Let ${ }^{-}: k[G] \rightarrow k[G]$ be the canonical involution of the group ring $k[G]$, in other words the $k$-linear involution of $k[G]$ characterized by $\bar{g}=g^{-1}$ for all $g \in G$. Let $U_{G}$ be the linear algebraic group defined over $k$ such that for every commutative $k$-algebra $A$, we have $U_{G}(A)=\{x \in A[G] \mid x \bar{x}=1\}$. Recall that we denote by $H^{1}\left(k, U_{G}\right)$ the pointed set $H^{1}\left(\Gamma_{k}, U\left(k_{s}\right)\right)$.

Let $f_{L}: \Gamma_{k} \rightarrow G$ be a continuous homomorphism corresponding to $L$. The composition of $f_{L}$ with the inclusion of $G$ in $U_{G}\left(k_{s}\right)$ is a 1 -cocycle $\Gamma_{k} \rightarrow U_{G}\left(k_{s}\right)$. Let us denote by $u(L)$ its class in the cohomology set $H^{1}\left(k, U_{G}\right)$. The following is proved in [5], Proposition 1.5.1: 
PROPOSITION 2.6. Let $L$ and $L^{\prime}$ be two G-Galois algebras. Then the $G$-quadratic forms $q_{L}$ and $q_{L^{\prime}}$ are isomorphic if and only if $u(L)=u\left(L^{\prime}\right) \in H^{1}\left(k, U_{G}\right)$.

The trace form of a $G$-Galois algebra, considered as a $G$-form, determines the trace forms of all of its subalgebras of fixed points (see [5], Section 1.4). We have a similar result for multiples of trace forms, as follows:

PROPOSITION 2.7. Let $L$ and $L^{\prime}$ be two $G$-Galois algebras. Let $H$ be a subgroup of $G$, and set $E=L^{H}, E^{\prime}=L^{\prime H}$. Let $\phi$ be a quadratic form over $k$. Suppose that $\phi \otimes q_{L} \simeq_{G} \phi \otimes q_{L^{\prime}}$. Then we have (a) The quadratic forms $\phi \otimes q_{E}$ and $\phi \otimes q_{E^{\prime}}$ are isomorphic. (b) If moreover $H$ is a normal subgroup of $G$, then the $(G / H)$-quadratic forms $\phi \otimes q_{E}$ and $\phi \otimes q_{E^{\prime}}$ are isomorphic.

PROOF. The proof of this statement is similar to the proof of 1.5.1, in [5]. The homomorphism $f_{L}: \Gamma_{k} \rightarrow G$ induces $f_{L}^{*}: H^{1}(G, \mathbf{Z} / 2 \mathbf{Z}) \rightarrow H^{1}(k)$. For any $x \in H^{1}(G, \mathbf{Z} / 2 \mathbf{Z})$, set $x_{L}=f_{L}^{*}(x)$. Then the elements $x_{L}$ are invariants of the $G$-quadratic form $q_{L}$ (cf. [5], 2.2.3). Let $x \in H^{1}(G, \mathbf{Z} / 2 \mathbf{Z})$, and let $\chi: G \rightarrow \mathbf{Z} / 2 \mathbf{Z}$ the corresponding homomorphism. Let $H$ be the kernel of $\chi$, and let $E_{\chi}=L^{H}$ be the invariant subalgebra; it is a quadratic subalgebra of $L$. The discriminant of the quadratic algebra $E_{\chi}$ is equal to $x_{L}$.

The following is a generalization of [5], 2.2.3:

PROPOSITION 2.8. Let $L$ and $L^{\prime}$ be two $G$-Galois algebras. Let $\phi \in I^{d-1}$. Suppose that $\phi \otimes q_{L} \simeq_{G} \phi \otimes q_{L^{\prime}}$. Then $e_{d-1}(\phi) \cup x_{L}=e_{d-1}(\phi) \cup x_{L^{\prime}}$ for all $x \in H^{1}(G, \mathbf{Z} / 2 \mathbf{Z})$.

PROOF. Let $x \in H^{1}(G, \mathbf{Z} / 2 \mathbf{Z})$, and let $\chi: G \rightarrow \mathbf{Z} / 2 \mathbf{Z}$ the corresponding homomorphism. Let $H$ be the kernel of $\chi$, and set $E_{\chi}=L^{H}, E_{\chi}^{\prime}=L^{\prime H}$. By Proposition 2.7, the quadratic forms $\phi \otimes q_{E}$ and $\phi \otimes q_{E^{\prime}}$ are isomorphic. Using 2.4, we have $e_{d-1}(\phi) \cup \operatorname{disc}\left(q_{E}\right)=e_{d-1}(\phi) \cup \operatorname{disc}\left(q_{E^{\prime}}\right)$. As the discriminant of a quadratic algebra is equal to the discriminant of its trace form, we obtain $e_{d-1}(\phi) \cup x_{L}=e_{d-1}(\phi) \cup x_{L^{\prime}}$, and so the proposition is proved.

\subsection{Hermitian forms over division algebras with involution}

Let $D$ be a division algebra over $k$. An involution of $D$ is a $k$-linear anti-automorphism $\sigma: D \rightarrow D$ of order 2 . Let $K$ be the center of $D$. We say that $(D, \sigma)$ is a division algebra with involution over $k$ if the fixed field of $\sigma$ in $K$ is equal to $k$. If $K=k$, then $\sigma$ is said to be of the first kind. After extension to $k_{s}$, the involution $\sigma$ is determined by a symmetric or a skew-symmetric form. In the first case, $\sigma$ is said to be of the orthogonal type, and in the second one, of the symplectic type. If $K \neq k$, then $K$ is a quadratic extension of $k$ 
and the restriction of $\sigma$ to $K$ is the non-trivial automorphism of $K$ over $k$. In that case, the involution is said to be of the second kind, or a unitary involution, or a $K / k$-involution. See for instance [11] or [15], ch 7, for more details on algebras with involution. Let $(D, \sigma)$ be a division algebra with involution over $k$. A hermitian form over $(D, \sigma)$ is by definition a pair $(V, h)$, where $V$ is a finite dimensional $D$-vector space, and $h: V \times V \rightarrow D$ is hermitian with respect to $\sigma$. We say that $(V, h)$ is hyperbolic if there exists a sub $D$-vector space $W$ of $V$ with $\operatorname{dim}(V)=2 \operatorname{dim}(W)$ and such that $h(x, y)=0$ for all $x, y \in W$. This leads to a notion of Witt group $W(D, \sigma)$ (cf. for instance [15], Ch 7. $\S 2$ ). Note that the tensor product of a quadratic form over $k$ with a hermitian form over $(D, \sigma)$ is a hermitian form over $(D, \sigma)$, hence $W(D, \sigma)$ is a $W(k)$-module. Let $(V, h)$ be a hermitian form over $(D, \sigma)$, as above. Let $n=\operatorname{dim}_{D}(V)$, and let $H$ be the matrix of $h$ with respect to some $D$-basis of $V$. Let us denote by $\mathrm{Nrd}: M_{n}(D) \rightarrow k$ the reduced norm. The discriminant of $h$ is by definition $\operatorname{disc}(h)=(-1)^{\frac{n(n-1)}{2}} \operatorname{Nrd}(H) \in k^{*} / k^{* 2}$.

\section{Multiples of Hermitian Forms}

Let $(D, \sigma)$ a division algebra with involution over $k$. Let us denote by $J$ the sub $W(k)-$ module of $W(D, \sigma)$ consisting of the hermitian forms $(V, h)$ with $\operatorname{dim}_{D}(V)$ even. Suppose that $\operatorname{cd}_{2}\left(\Gamma_{k}\right) \leq d$.

THEOREM 3.1. (a) We have $I^{d} J=0$.

(b) If $\sigma$ is of the second kind, then $I^{d-1} J=0$.

(c) If $\sigma$ is of the first kind and of the symplectic type, then $I^{d-2} J=0$.

Part (a) was proved by Chabloz in [9]. We need the following lemma:

LEMMA 3.2. Let $a \in D^{*}$ such that $\sigma(a)=a$. We have:

(a) If $\phi \in I^{d}$, then $\phi \otimes<1, a>$ is hyperbolic.

(b) If $\phi \in I^{d-1}$ and $\sigma$ is of the second kind, then $\phi \otimes<1, a>$ is hyperbolic.

(c) If $\phi \in I^{d-2}$ and $\sigma$ is of the first kind and of the symplectic type, then $\phi \otimes<1, a>$ is hyperbolic.

PROOF.

(a) Let $F=k(a)$, and let $f: W(F) \rightarrow W(D, \sigma)$ be the base change homomorphism. We have $f(\phi \otimes<1, a>)=\phi \otimes<1, a>$, and hence it suffices to check that

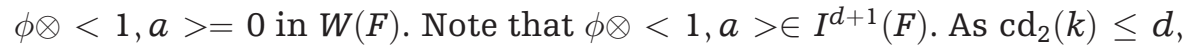


we have $\operatorname{cd}_{2}(F) \leq d$, therefore by Theorem 2.1, we have $I^{d+1}(F)=0$. Hence $\phi \otimes<1, a>$ is hyperbolic, and this concludes the proof of (a).

(b) Suppose that $\sigma$ is a $K / k$-involution, and set $E=K(a), F=k(a)$. Let us denote by $\tau: E \rightarrow E$ the restriction of $\sigma: D \rightarrow D$ to $E$. Then $\tau$ is an $E / F$-involution, an involution of the second kind. Let $f: W(E, \tau) \rightarrow W(D, \sigma)$ be the base change homomorphism. We have $f(\phi \otimes<1, a>)=\phi \otimes<1, a>$, hence it suffices to check that $\phi \otimes\langle 1, a\rangle=0$ in $W(E)$. Let $E=F(\sqrt{\delta})$, for some $\delta \in F$. Let $\operatorname{tr}_{E / F}: W(E, \tau) \rightarrow W(F)$ be the $W(F)$-homomorphism given by the trace of hermitian forms. It is well-known that $\operatorname{tr}_{E / F}$ is injective, and its image is equal to $\langle 1,-\delta>W(F)$ (cf. for instance [15], Ch 10, $\S 1$ ). We have

$\left.\operatorname{tr}_{E / F}(<1, a>)\right)=<1,-\delta>\otimes<1, a>$,

hence $\operatorname{tr}_{E / F}(\phi \otimes<1, a>)=\phi \otimes<1,-\delta>\otimes<1, a>$. This implies that

$\operatorname{tr}_{E / F}(\phi \otimes<1, a>) \in I^{d+1}(F)$.

As in (a), we see that $I^{d+1}(F)=0$. Therefore, $\phi \otimes<1, a>=0$ in $W(E, \tau)$, and $(\mathrm{b})$ is proved.

(c) Suppose that $\sigma$ is symplectic. Then the degree of $D$ is even. Set $\operatorname{deg}(D)=2 m$, and let us prove the statement by induction on $m$. If $m=1$, then $D$ is a quaternion algebra and $\sigma$ is the canonical involution of $D$. As $\sigma(a)=a$, we have $a \in k^{*}$. Let $\operatorname{trd}: W(D, \sigma) \rightarrow W(k)$ be the $W(k)$-homomorphism given by the reduced trace of hermitian forms. It is well known that this homomorphism is injective, and its image is equal to $n_{D} W(k)$, where $n_{D}$ is the norm form of the quaternion algebra $D$ (cf. [15], ch. 10, $\S 1$ ). We have $\operatorname{trd}(<1, a>)=n_{D} \otimes<1, a>\in I^{3}$. Let $\phi \in I^{d-2}$. Then

$\operatorname{trd}(\phi \otimes<1, a>)=\phi \otimes n_{D} \otimes<1, a>\in I^{d+1}=0$.

This implies that $\operatorname{trd}(\phi \otimes<1, a>)=0$ in $W(k)$, hence $\phi \otimes<1, a>=0$ in $W(D, \sigma)$. Suppose that $m>1$. If $a \notin k$, set $F=k(a)$. If $a \in k$, take any $b \in D^{*}$ such that $\sigma(b)=b$ and that $b \notin k$ (this is possible as $m>1$ ) and set $F=k(b)$. Let $D^{\prime}=Z_{D}(F)$ be the centralizer of $F$ in $D$. Note that $F$ is invariant by $\sigma$. By $[11], 2.9$. we know that $[F: k] \leq m$. The $F$-algebra 
$D^{\prime}$ is also invariant by $\sigma$. We have $[F: k]>1$, hence $\operatorname{deg} D^{\prime}<\operatorname{deg}(D)$. Let $\operatorname{deg}\left(D^{\prime}\right)=2 m^{\prime}$. As $m^{\prime}<m$, we can apply the induction hypothesis, hence $\phi \otimes<1, a>=0$ in $W\left(D^{\prime}, \sigma\right)$. Let $f: W\left(D^{\prime}, \sigma\right) \rightarrow W(D, \sigma)$ be the base change homomorphism. We have $f(\phi \otimes<1, a>)=\phi \otimes<1, a>$. Hence $\phi \otimes<1, a>=0$ in $W(D, \sigma)$, as claimed.

PROOF OF THEOREM 3.1. Let $q \in J$. We have $q=<a_{1}, \ldots, a_{n}>$, with $a_{i} \in D^{*}, \sigma\left(a_{i}\right)=a_{i}$. Note that $n$ is even, as $q \in J$. Set $m=\frac{n}{2}$. Let $H=<1,-1>$, and let us denote by $[m] H$ the orthogonal sum of $m$ copies of $H$. Then in $W(D, \sigma)$, we have $q=q \oplus[m] H=<1, a_{1}>\oplus<$ $-1, a_{2}>\oplus \cdots \oplus<-1, a_{n}>$. By the lemma, $\phi \otimes<1, a_{i}>$ and $\phi \otimes<-1, a_{i}>$ are hyperbolic for all $i$ whenever $\phi \in I^{d}$, or $\phi \in I^{d-1}$ and $\sigma$ is unitary, or $\phi \in I^{d-2}$ and $\sigma$ is symplectic. Hence $\phi \otimes q$ is hyperbolic in these cases too, so the theorem is proved.

The following is a consequence of results of Parimala, Sridharan and Suresh [14] and of Berhuy [8].

THEOREM 3.3. Suppose that $D$ is a quaternion algebra, and that $\sigma$ is of the first kind and of the orthogonal type. Let $h \in J$, and let $\phi \in I^{d-1}$. Then $\phi \otimes h$ is hyperbolic if and only if $e_{d-1}(\phi) \cup(\operatorname{disc}(h))=0$.

Proof. By Berhuy [8], Th. 13, it suffices to show that $e_{d-1}(\phi) \cup(\operatorname{disc}(h))=0$ if and only if $e_{n, D}(\phi \otimes h)=0$ for all $n \geq 0$ (cf. [8], 2.2 for the definition of the invariant $\left.e_{n, D}\right)$. As $\operatorname{cd}_{2}\left(\Gamma_{k}\right) \leq d$, we have $e_{n, D}(\phi \otimes h)=0$ for $n>d$, so it suffices to check that $e_{d-1}(\phi) \cup(\operatorname{disc}(h))=0$ is equivalent with $e_{n, D}(\phi \otimes h)=0$ for all $n=0, \ldots, d$. Let $k(D)$ be the function field of the quadric associated to $D$. Then $D \otimes k(D) \simeq M_{2}(k(D))$, and $h_{k(D)}$ corresponds, via Morita equivalence, to a quadratic form $q_{h}$ over $k(D)$. Note that $\operatorname{disc}\left(q_{h}\right)=\operatorname{disc}(h)$. Similarly, the hermitian form $(\phi \otimes h)_{k(D)}$ corresponds to a quadratic form $q_{\phi h}$ over $k(D)$, and we have $q_{\phi h} \simeq \phi \otimes q_{h}$. For all $n=0, \ldots, d$, we have by construction that $e_{n, D}(\phi \otimes h)=0$ if and only if $e_{n}\left(q_{\phi h}\right)=0$ (cf. [8], 2.2). But $q_{\phi h} \simeq \phi \otimes q_{h}$ and hence

$$
e_{n}\left(q_{\phi h}\right)=e_{n}\left(\phi \otimes q_{h}\right)=e_{n-1}(\phi) \cup\left(\operatorname{disc}\left(q_{h}\right)\right)=e_{n-1}(\phi) \cup(\operatorname{disc}(h)) .
$$

If $n<d$, then $e_{n}(\phi)=0$ as $\phi \in I^{d-1}$. We have $e_{d}\left(q_{\phi h}\right)=e_{d-1}(\phi) \cup(\operatorname{disc}(h))$. Hence $e_{n}\left(q_{\phi h}\right)=0$ for all $n \geq 0$ if and only if $e_{d-1}(\phi) \cup(\operatorname{disc}(h))=0$. This concludes the proof.

COROLLARY 3.4. Suppose that $D$ is a quaternion algebra, and that $\sigma$ is of the first kind and of the orthogonal type. Let $h$ and $h^{\prime}$ be two hermitian forms over $(D, \sigma)$, and let $\phi \in I^{d-1}$. Then $\phi \otimes h \simeq \phi \otimes h^{\prime}$ if and only if $e_{d-1}(\phi) \cup(\operatorname{disc}(h))=e_{d-1}(\phi) \cup\left(\operatorname{disc}\left(h^{\prime}\right)\right)$. 
PROOF. The hermitian forms $\phi \otimes h$ and $\phi \otimes h^{\prime}$ are isomorphic if and only if $\phi \otimes\left(h \oplus\left(-h^{\prime}\right)\right)$ is hyperbolic. By Theorem 3.3, this is equivalent with $e_{d-1}(\phi) \cup \operatorname{disc}\left(h \oplus\left(-h^{\prime}\right)\right)=0$. Note that as $\operatorname{dim}_{k}(D)$ is even, $\operatorname{disc}\left(-h^{\prime}\right)=\operatorname{disc}\left(h^{\prime}\right)$. Therefore

$$
e_{d-1}(\phi) \cup\left(\operatorname{disc}\left(h \oplus\left(-h^{\prime}\right)\right)\right)=e_{d-1}(\phi) \cup(\operatorname{disc}(h))+e_{d-1}(\phi) \cup\left(\operatorname{disc}\left(h^{\prime}\right)\right),
$$

and hence the corollary is proved. Let us denote by $J_{2}$ the sub $W(k)$-module of $J$ consisting of the classes of the hermitian forms $h$ such that $(\operatorname{disc}(h))=0$.

COROLLARY 3.5. Suppose that $D$ is a quaternion algebra, and that $\sigma$ is of the first kind and of the orthogonal type. Then $I^{d-1} J_{2}=0$.

PROOF. This is an immediate consequence of Corollary 3.4

\section{Multiples of Trace Forms}

Let $L$ and $L^{\prime}$ be two G-Galois algebras. The aim of this section is to prove a result concerning multiples of trace forms (see Corollary 4.2) that was conjectured in [1], and to derive some consequences for generalized self-dual normal bases. Suppose that $\operatorname{cd}_{2}\left(\Gamma_{k}\right) \leq d$.

THEOREM 4.1. Let $\phi \in I^{d-1}$. Then $\phi \otimes q_{L} \simeq_{G} \phi \otimes q_{L^{\prime}}$ if and only if $e_{d-1}(\phi) \cup x_{L}=e_{d-1}(\phi) \cup x_{L^{\prime}}$ for all $x \in H^{1}(G, \mathbf{Z} / 2 \mathbf{Z})$.

Special cases of this have been proved in [1], [4], [5], [9], [12] and [16].

PROOF. The condition is necessary by Proposition 2.8. Let us prove that it is also sufficient. By [3], 4.1 and [5], 2.3.1 we can assume that $k$ is perfect. Set $A=k[G]$, and let us denote by $\sigma_{A}: A \rightarrow A$ the canonical involution. Let $R_{A}$ be the radical of the algebra $A$, and set $\bar{A}=A / R$. Then the projection $A \rightarrow \bar{A}$ induces a bijection of pointed sets $H^{1}\left(k, U_{A}\right) \rightarrow H^{1}\left(k, U_{\bar{A}}\right)$. We have

$$
\bar{A} \simeq A_{1} \times \cdots \times A_{s} \times\left(A_{s+1} \times A_{s+1}^{\prime}\right) \times \cdots \times\left(A_{m} \times A_{m}^{\prime}\right)
$$

where $A_{i}$ is a simple algebra for all $i=1, \ldots, m$, with $\sigma\left(A_{i}\right)=A_{i}$ for $i=1, \ldots, s$ and $\sigma\left(A_{i}\right)=A_{i}^{\prime}$ for $i=s+1, \ldots, m$. Let $\sigma_{i}: A_{i} \rightarrow A_{i}$ be the restriction of $\sigma_{A}$ to $A_{i}$ for $i=1, \ldots, s$, and let us denote by $\sigma_{i}: A_{i} \times A_{i}^{\prime} \rightarrow A_{i} \rightarrow A_{i} \times A_{i}^{\prime}$ the restriction of $\sigma_{A}$ to $A_{i} \times A_{i}^{\prime}$ if $i=s+1, \ldots, m$. Let $F_{i}$ be the maximal subfield of the center of $A_{i}$ such that $\sigma_{i}$ is $F_{i}$-linear if $i=1, \ldots, s$, and let $U_{i}$ be the norm-one-group of $\left(A_{i}, \sigma_{i}\right)$. For $i=s+1, \ldots, m$, let $F_{i}$ be 
the center of $A_{i}$, and let $U_{i}$ be the norm-one-group of $\left(\left(A_{i} \times A_{i}\right), \sigma_{i}\right)$. Then $U_{i}$ is a linear algebraic group defined over $F_{i}$ for all $i=1, \ldots, m$. We have a bijection of pointed sets

$$
H^{1}\left(k, U_{A}\right) \rightarrow \prod_{i=1, \ldots, m} H^{1}\left(F_{i}, U_{i}\right)
$$

If $i=s+1, \ldots, m$, then $U_{i}$ is a general linear group, hence $H^{1}\left(F_{i}, U_{i}\right)=0$. Hence we have a bijection of pointed sets

$$
H^{1}\left(k, U_{A}\right) \rightarrow \prod_{i=1, \ldots, S} H^{1}\left(F_{i}, U_{i}\right)
$$

Let us denote by $u_{i}, u_{i}^{\prime} \in H^{1}\left(F_{i}, U_{i}\right), i=1, \ldots, s$, the images of $u(L), u\left(L^{\prime}\right) \in H^{1}\left(k, U_{A}\right)$. For all $i=1, \ldots, s$, the simple algebra $A_{i}$ is a matrix algebra over a division algebra with involution $D_{i}$, and the classes $u_{i}, u_{i}^{\prime}$ correspond to isomorphism classes of hermitian forms $h_{i}, h_{i}^{\prime}$ over $D_{i}$.

Let $r=\operatorname{dim}(\phi)$, and set $B=M_{r}(A)$. Let us denote by $\sigma_{B}: B \rightarrow B$ the involution induced by $\sigma_{A}$ and the transposition, i.e. $\sigma_{B}\left(a_{i j}\right)=\left(\sigma_{A}\left(a_{j, i}\right)\right)$ for all $a_{i, j} \in A$. Let $R_{B}$ be the radical of $B$, and set $\bar{B}=B / R_{B}$.

We have

$$
\bar{B} \simeq M_{r}\left(A_{1}\right) \times \cdots \times M_{r}\left(A_{m}\right)
$$

As above, we get a bijection of pointed sets

$$
H^{1}\left(k, U_{B}\right) \rightarrow \prod_{i=1, \ldots, S} H^{1}\left(F_{i}, U_{M_{r}\left(A_{i}\right)}\right)
$$

Sending a $G$-quadratic form to its tensor product with the quadratic form $\phi$ gives us a map $f: H^{1}\left(k, U_{A}\right) \rightarrow H^{1}\left(k, U_{B}\right)$. The map $f$ induces $\bar{f}: H^{1}\left(k, U_{\bar{A}}\right) \rightarrow H^{1}\left(k, U_{\bar{B}}\right)$, and

$$
f_{i}: H^{1}\left(F_{i}, U_{i}\right) \rightarrow H^{1}\left(k, U_{M_{r}\left(A_{i}\right)}\right)
$$

for all $i=1, \ldots, s$. The image of the isomorphism class of the hermitian form $h_{i}$ is the hermitian form $\phi \otimes h_{i}$.

Let us show that for all $i=1, \ldots, s$, we have $\phi \otimes h_{i} \simeq \phi \otimes h_{i}^{\prime}$. This is equivalent to proving that $\phi \otimes\left(h_{i} \oplus\left(-h_{i}^{\prime}\right)\right)$ is hyperbolic. If $U_{i}$ is unitary or symplectic, then this follows from Theorem 3.1. (b) and (c). Suppose that $U_{i}$ is orthogonal. Then $A_{i}=M_{n_{i}}\left(D_{i}\right)$, 
where $D_{i}=F_{i}$ or $D_{i}$ is a quaternion field with center $F_{i}$ (cf. [15], Ch. 8, 13.5. (ii)). We have $\left(U_{i} / U_{i}^{0}\right)\left(k_{s}\right) \simeq \mathbf{Z} / 2 \mathrm{Z}$. Let $\iota: \Gamma_{F_{i}} \rightarrow \Gamma_{k}$ be the inclusion, and let us consider

$$
\delta_{i}: \Gamma_{F_{i}} \stackrel{\iota}{\rightarrow} \Gamma_{k} \stackrel{\phi_{L}}{\longrightarrow} G \rightarrow U_{G}\left(k_{s}\right) \rightarrow U_{i}\left(k_{s}\right) \simeq \mathrm{Z} / 2 \mathrm{Z}
$$

and

$$
\delta_{i}^{\prime}: \Gamma_{F_{i}} \stackrel{\iota}{\rightarrow} \Gamma_{k} \stackrel{\phi_{L^{\prime}}}{\longrightarrow} G \rightarrow U_{G}\left(k_{s}\right) \rightarrow U_{i}\left(k_{s}\right) \simeq \mathbf{Z} / 2 \mathbf{Z} .
$$

Then $\delta_{i}, \delta_{i}^{\prime}$ are 1-cocycles that define elements $\left(\delta_{i}\right),\left(\delta_{i}^{\prime}\right) \in H^{1}\left(F_{i}, \mathbf{Z} / 2 \mathbf{Z}\right)$ corresponding to the relative discriminants $\operatorname{disc}\left(h_{i}\right)$ and $\operatorname{disc}\left(h_{i}^{\prime}\right)$ of the hermitian forms $h_{i}$ and $h_{i}^{\prime}$ with respect to the unit hermitian form $h_{0}$.

Note that $\delta_{i}=\iota X_{L}$ and $\delta_{i}=\iota x_{L^{\prime}}$ for all $x \in H^{1}(G, \mathbf{Z} / 2 \mathbf{Z})$. By hypothesis, we have $e_{d-1}(\phi) \cup x_{L}=e_{d-1}(\phi) \cup x_{L^{\prime}}$ for all $x \in H^{1}(G, \mathbf{Z} / 2 \mathbf{Z})$. This implies that

$$
e_{d-1}(\phi) \cup\left(\operatorname{disc}\left(h_{i}\right)\right)=e_{d-1}(\phi) \cup\left(\operatorname{disc}\left(h_{i}^{\prime}\right)\right)
$$

for all $i=1, \ldots, s$. By Corollary. 3.4, we conclude that the hermitian forms $\phi \otimes h_{i}$ and $\phi \otimes h_{i}^{\prime}$ are isomorphic, and hence the theorem is proved. Recall that for any $G$-quadratic form $q$, we denote by $[m] q$ the orthogonal sum of $m$ copies of $q$, in other words the quadratic form $\langle 1, \ldots, 1\rangle \otimes q$. Let us denote by $\epsilon_{d-1} \in H^{d-1}(k)$ the cup product of $d-1$ copies of $(-1) \in H^{1}(k)$. The following is an immediate consequence of Theorem 4.1:

COROLLARY 4.2. Let $L$ and $L^{\prime}$ be two $G-G a l o i s$ algebras. Then $\left[2^{d-1}\right] q_{L} \simeq_{G}\left[2^{d-1}\right] q_{L^{\prime}}$ if and only if $\epsilon_{d-1} \cup x_{L}=\epsilon_{d-1} \cup x_{L^{\prime}}$.

Let $L_{0}$ be the split $G$-Galois algebra, and let $q_{0}=q_{L_{0}}$ be its trace form. Recall that a $G$-Galois algebra is said to have a self-dual normal basis if $q_{L} \simeq_{G} q_{0}$. For any positive integer $m$, we denote by $[m] L$ the product of $m$ copies of the $G$-Galois algebra $L$. We say that $[m] L$ has a self-dual normal basis if $[m] q_{L} \simeq_{G}[m] q_{0}$. A subalgebra $E$ of $L$ is said to be a subalgebra of invariants if there exists a subgroup $H$ of $G$ such that $E=L^{H}$.

COROLLARY 4.3. Let $L$ be a G-Galois algebra. Then the algebra $\left[2^{d-1}\right] L$ has a self-dual normal basis if and only if the discriminant of every quadratic subalgebra of invariants is a sum of $2^{d-1}$ squares.

PRoOF. Let us denote by $q$ the $2^{d-1}$-dimensional unit form, and let $D(q)$ be the set of non-zero elements of $k$ represented by $q$. Then $a \in D(q)$ if and only if $a$ is a sum of $2^{d-1}$ squares in $k$. It is well known that $a \in D(q)$ if and only if the quadratic form $q \oplus<-a>$ is 
isotropic. This is equivalent to the $2^{d}$-fold Pfister form $q \otimes<1,-a>$ being hyperbolic; hence by 2.1 with $\epsilon_{d-1} \cup(a)=0$.

Let $x \in H^{1}(G, \mathbf{Z} / 2 \mathbf{Z})$. The argument above shows that $x_{L}$ is a sum of $2^{d-1}$ squares if and only if $\epsilon_{d-1} \cup x_{L}=0$. Let $\chi: G \rightarrow \mathbf{Z} / 2 \mathbf{Z}$ the corresponding homomorphism. Let $H$ be the kernel of $\chi$, and let $E_{X}=L^{H}$ be the invariant subalgebra. Then, $E_{X}$ is a quadratic subalgebra of $L$, and its discriminant is equal to $x_{L}$.

By Corollary 4.3 , the algebra $\left[2^{d-1}\right] L$ has a self-dual normal basis if and only if $\epsilon_{d-1} \cup X_{L}=0$ for all $x \in H^{1}(G, \mathbf{Z} / 2 \mathbf{Z})$. We have just seen that this is equivalent to the discriminant of the quadratic subalgebra $E_{X}$ being a sum of $2^{d-1}$ squares for all $x \in H^{1}(G, \mathbf{Z} / 2 \mathbf{Z})$; hence the corollary is proved.

\section{Acknowledgments}

This work was partially supported by the Swiss National Science Foundation, grant 200020-109174/1.

\section{References}

[1] Bayer-Fluckiger, E. "Galois cohomology and the trace form." Jahresbericht der Deutschen Mathematiker-Vereinigung 96 (1994): 35-55.

[2] _ "Self-Dual Normal Bases and Related Topics." Proceedings of the Conference Finite Fields and Applications (Augsburg, 1999), 25-36 Springer Verlag, 2001.

[3] Bayer-Fluckiger, E. and H. W. Lenstra, Jr. “Forms in odd degree extensions and self-dual normal bases." American Journal of Mathematics 112 (1990): 359-73.

[4] Bayer-Fluckiger, E., M. Monsurro, R. Parimala, and R. Schoof. "Trace forms of Galois algebras over fields of cohomological dimension $\leq$ 2." Pacific Journal of Mathematics 217 (2004): 29-43.

[5] Bayer-Fluckiger, E. and J. P. Serre. "Torsions quadratiques et bases normales autoduales." American Journal of Mathematics 116 (1994): 1-64.

[6] Bayer-Fluckiger, E. and M. Monsurro. "Doubling trace forms." St. Petersburg Mathematical Journal 11 (2000): 405-17.

[7] Bayer-Fluckiger, E. and J. Morales. "Multiples of trace forms in number fields." AMS Proceedings of Symposia in Pure Mathematics 58.2 (1995): 73-81.

[8] Berhuy, G. "Cohomological invariants of quaternionic skew-hermitian forms." Archiv Der Mathematik (forthcoming).

[9] Chabloz, Ph. "Anneau de Witt des $G$-formes et produit des $G$-formes trace par des formes quadratiques." Journal of Algebra 266 (2003): 338-61.

[10] Kahn, B. “La conjecture de Milnor, d'après Voevodsky, Séminaire Bourbaki (1996/97), Exp. 834 (juin 1997)." Astérisque 245 (1997): 379-418. 
[11] Knus, M., A. Merkurjev, M. Rost, and J. P. Tignol. The Book of Involutions, Vol. 44: Providence, RI: AMS Colloquium Publications, 1998.

[12] Monsurro, M. "Pfister forms times trace forms." Communications in Algebra 30 (2002): 3391-402.

[13] Orlov, D., A. Vishik, and V. Voedovsky. An exact sequence for Milnor's K-theory with applications to quadratic forms. (2001): preprint arxiv.org/abs/math/0101023.

[14] Parimala, R., R. Sridharan, and V. Suresh. "Hermitian analogue of a theorem of Springer." Journal of Algebra 243 (2001): 780-9.

[15] Scharlau, W. Quadratic and Hermitian Forms, Grundlehren der. Mathematischen Wissenschaften, Berlin: Springer-Verlag, 1985.

[16] Serre, J.-P. Cohomologie Galoisienne, Lecture Notes in Mathematics. Berlin: Springer-Verlag, (1964 and 1994).

[17] Corps Locaux. Paris: Hermann, 1968.

[18] - "Reduced power operations in motivic cohomology." Publications Mathématiques of the Institut des Hautes Études Scientifiques 98 (2003): 1-57.

[19] "Motivic cohomology with Z/2Z-coefficients." Publications Mathématiques of the Institut des Hautes Études Scientifiques 98 (2003): 59-104. 